\title{
Design of 3D-Printed Nasopharyngeal Swabs for Children is Enabled by Radiologic Imaging
}

\author{
(D) Z. Starosolski, DP. Admane, (D). Dunn, (D) B. Kaziny, (D)T.A.G.M. Huisman, and (D)A. Annapragada
}

\section{ABSTRACT}

SUMMARY: 3D-printed nasopharyngeal swabs for COVID-19 molecular diagnostic testing address the national shortage of swabs. Swab designs for adult use were placed in the public domain in March 2020. Swabs for pediatric use, however, need to be smaller and more flexible to navigate delicate pediatric nasopharyngeal cavities. We describe a novel use of maxillofacial CT scans to aid in the design of pediatric nasopharyngeal swabs.

ABBREVIATIONS: COVID-19 = coronavirus disease 2019; RT-PCR = real-time polymerase chain reaction

C oronavirus disease 2019 (COVID-19) is a worldwide pandemic and has resulted in shortages of medical supplies, including nasopharyngeal swabs used in diagnostic tests. 3D printing provides a novel solution, and designs for 3D-printed nasopharyngeal swabs are freely distributed via a GitHub repository. ${ }^{1}$ The effort, however, has focused on swabs for use in adult patients. These adult swabs are too inflexible and too large for safe pediatric use, particularly in children younger than 3 years of age. At our hospital (Texas Children's Hospital), a large pediatric tertiary-care center, a 3D-printed swab that replicated the dimensions of a commercial pediatric swab (COPAN Flock Technologies; Puritan Diagnostics) was evaluated by one of our pediatric emergency physicians. However, the $3 \mathrm{D}$-printed swab was not sufficiently flexible and would risk damaging the nasal passages of the child. Simply reducing the diameter of the flexible shaft was deemed inadvisable because the use of the swab involves twisting in both directions after insertion and a thinner shaft would risk breakage in situ. We, therefore, set out to design and produce novel 3D-printed swabs for use in infants and young children.

Received July 6, 2020; accepted after revision July 22.

From the Edward B. Singleton Department of Radiology (Z.S., T.A.G.M.H., A.A.) Texas Children's Hospital, Houston, Texas; Department of Radiology (P.A.), Baylor College of Medicine, Houston, Texas; and Departments of Pediatrics (B.K.), and Medical Microbiology and Virology (J.D.), Texas Children's Hospital, Houston,

Texas.

This work was partially funded by a grant from the Soicher Family Foundation to A.A.

Please address correspondence to Ananth Annapragada, PhD, Edward B. Singleton Department of Radiology, Texas Children's Hospital. 1102 Bates St Suite 850, Feigin Center, Houston, TX 77030; e-mail: avannapr@texaschildrens.org

- Indicates open access to non-subscribers at www.ajnr.org

http://dx.doi.org/10.3174/ajnr.A6794

\section{MATERIALS AND METHODS}

The institutional review board approved all clinical data use. Examination of maxillofacial CT scans of patients 1-3 years of age suggested that primary deflection of the swab in the sagittal plane would be sufficient to navigate from the external nares to the posterior nasopharynx. We, therefore, designed elliptical cross-section swabs (Fig 1) and verified that sagittal shaft deflection was adequate. We then made 3D prints of the nasopharyngeal passage itself, and physically tested the ability of individual swab prototypes to navigate the passage. We noted that less flexible designs would get trapped in the crenulations of the nasopharyngeal passage and might require multiple attempts to reach the posterior nasopharynx, thus lengthening the time required to reach the sampling location and possibly injuring the delicate pediatric anatomy. On the assumption that increased navigation time and resistance correlated with patient discomfort, we chose the navigation time and a qualitative assessment of resistance as measures of patient discomfort, ultimately selecting the design with the shortest navigation time and the lowest resistance score for clinical use.

Maxillofacial CT datasets of 5 patients, 11 to 34 months of age and unremarkable for pathologies involving the maxillofacial region, were randomly selected from our data base. Images were processed in 3D Slicer (Version 4.10; http://www.slicer.org) to create Standard Triangle Language files for printing. Nasopharyngeal passages were printed on a Form2 printer using Elastic Resin (Formlabs). Swab designs were made in Fusion 360 (AUTODESK) and printed on a Form 2 printer using Surgical Guide Resin (Formlabs).

We tested 5 swabs designs: 2 commercial mini-swabs, Flocked 1, PURITAN Diagnostics and Flocked 2, COPAN Flock Technologies; and 3 in-house-printed, all of which had a flexible shaft length of approximately $50 \mathrm{~mm}$, a handle of $78 \mathrm{~mm}$, and a transition zone of 

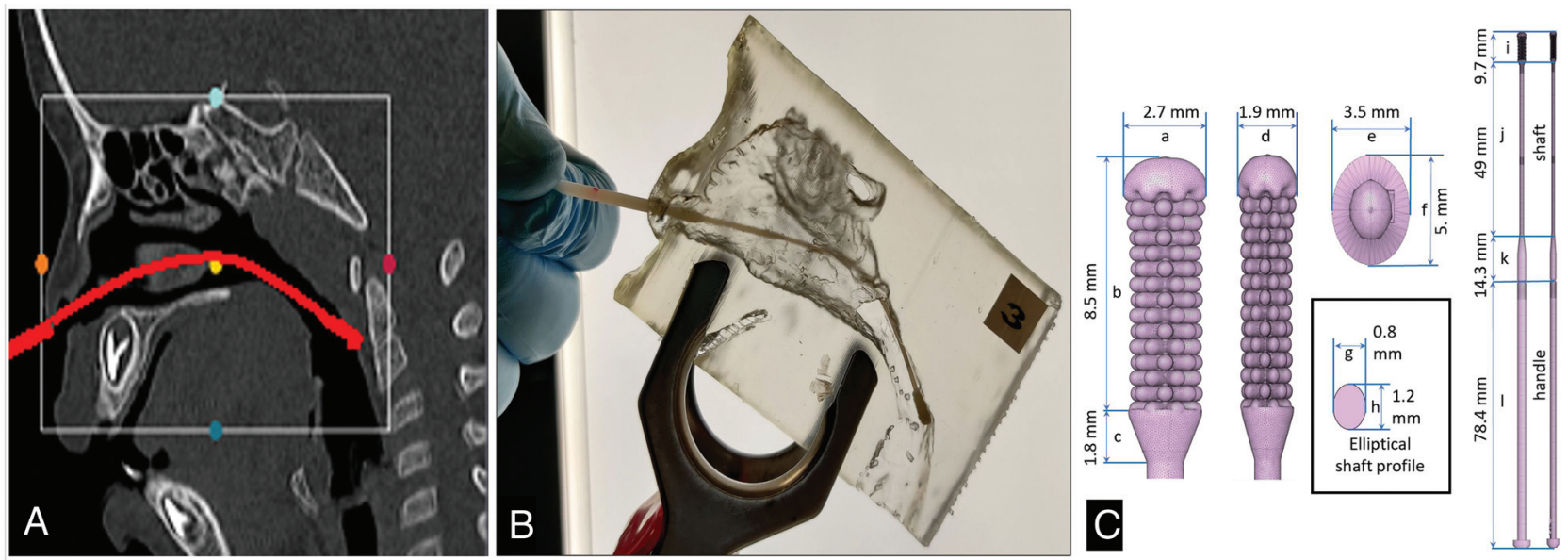

FIG 1. A, Sagittal section of a maxillofacial CT scan of a 22-month-old patient. Shown in red is the trajectory of a virtually inserted swab reaching the posterior nasopharynx. The white box shows the portion of the anatomy chosen to print the nasopharyngeal passage. $B, A$ commercial flocked pediatric swab (COPAN Flock Technologies swab) reaching the posterior nasopharynx within the 3D-printed nasopharyngeal passage. $C$, Design of the elliptical-section 3D-printed pediatric swab (Design ES).

Tabularized dimensions for 2 commercial and 3 in-house printed swab designs ${ }^{a}$

\begin{tabular}{lcccccccccccc}
\hline \multicolumn{1}{c}{ Swab } & a & b & c & d & e & f & g & h & i & j & k & l \\
\hline Flocked 1 & 2.3 & 7.0 & 0 & 2.3 & 2.5 & 2.5 & 1.2 & 1.2 & 7.0 & 44 & 8.3 & 94 \\
Flocked 2 & 2.7 & 7.4 & 0 & 2.7 & 2.5 & 2.5 & 0.9 & 0.9 & 7.4 & 66 & 24.5 & 53 \\
Design E & 2.7 & 8.5 & 0 & 1.9 & 3.5 & 5.0 & 0.8 & 1.2 & 8.5 & 50.8 & 14.3 & 78.4 \\
Design I & 2.7 & 8.5 & 0 & 2.6 & 5.0 & 5.0 & 1.2 & 1.2 & 8.5 & 50.8 & 14.3 & 78.4 \\
Design ES & 2.7 & 8.5 & 1.8 & 1.9 & 3.5 & 5.0 & 0.8 & 1.2 & 10.4 & 49 & 14.3 & 78.4 \\
\hline
\end{tabular}

${ }^{a}$ Dimensions are in millimeters. Columns represents dimensions as in Fig $1 \mathrm{C}$.

Syndrome coronavirus 2 nucleocapsid region and 1 target in the human RNase $\mathrm{P}$ gene to assess the cellular quality of the sample.

\section{RESULTS}

Two commercial flocked mini-swabs (from Puritan Diagnostics and COPAN Flock Technologies) and 3 in-house printed swab designs were tested. All

$14 \mathrm{~mm}$, as shown in Fig 1, and varied in the flexible shaft cross-section: Design E, $1.2 \mathrm{~mm} \times 0.8 \mathrm{~mm} \varnothing$; Design I, $1.2 \mathrm{~mm}$; and Design ES, $1.2 \mathrm{~mm} \times 0.8 \mathrm{~mm} \varnothing$, with an additional slanted posterior edge of the brush. The Table shows dimensions (in millimeters) for each of the swab types tested. Navigation of each swab from the external nares to the posterior nasopharynx was done by 1 individual, the order of testing of the individual swabs and anatomic models was randomized, and each test was repeated 3 times. The time to complete navigation was timed by an observer using a hand-held stopwatch, while the resistance on insertion was classified using a 3-level resistance score (1, easy insertion: no resistance; 2 , medium: mild resistance; and 3, hard: resistance requiring extra force). All statistical calculations were performed in Matlab R19a (MathWorks).

The quality of specimen recovery from Design ES nasopharyngeal swabs was determined by performing a crossover collection study with the commercial swabs (Puritan Diagnostics) and prototype NB swabs in 2 healthy adult volunteers, a 26-year-old man and a 28-year-old woman. Both nostrils of the volunteers were sampled by each swab in successive order. The swabs were placed into a MicroTest M4RT viral transport containing $3 \mathrm{~mL}$ of media (Remel). Nucleic acid was extracted using $200 \mathrm{uL}$ from each viral transport on the eMAG system (bioMérieux) and eluted into $50 \mathrm{uL}$ of buffer. Real-time polymerase chain reaction (RT-PCR) was performed on each eluate using the Centers for Disease Control's 2019Novel Coronavirus (2019-nCoV) Emergency Use Authorization assay, $^{2}$ which includes 2 targets in the Severe Acute Respiratory in-house swabs were successfully navigated to the posterior nasopharynx in all 5 nasopharyngeal passage models, while 1 commercial swab (COPAN Flock Technologies) was incapable of insertion into anatomic model 1 (Fig 2). On the basis of the shortest navigation time and the lowest resistance score (Fig 2C), Design ES was chosen as the preferred design for clinical testing. Preliminary clinical testing in 2 adult volunteers demonstrated recovery of cellular material from Design ES swabs to be within a concentration of 0.3 $\log$ of that from the currently used nasopharyngeal swabs.

\section{DISCUSSION}

Pediatric nasopharyngeal swabs (mini-swabs) are a thinner, smaller, more flexible version of those used for adults and are in even shorter supply during the COVID-19 pandemic than the adult swabs.

3D-printed swabs are fundamentally different from existing commercial swab designs in the form of the swab tip: While commercial swabs have flocked fibrous tips, 3D-printed swabs have solid tips with hemispheric nubs. However, both in vitro and in vivo testing have shown them to be practically identical in sample transfer for viral assay by RT-PCR both in our own labs and in other labs. ${ }^{3,4}$ Of note, at the time of this writing, the pediatric swabs printed using Design ES have now been used in our hospital in $>1000$ patients with no reported problems.

This brief report has several limitations. Only 5 nasopharyngeal prints of patients in a limited age range (1-3 years) were 

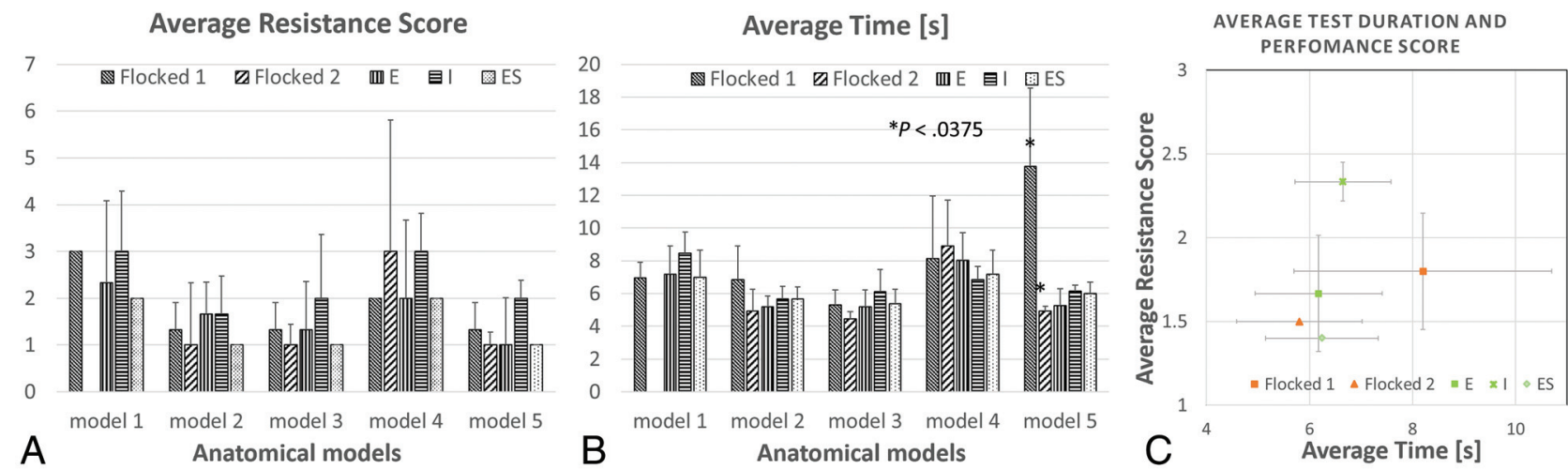

FIG 2. Results comparison based on 3 repetitions and 5 anatomic models. $A$, Average performance score (a lower value of the performance is better). $B$, Average time of the procedure in seconds. The Flocked 2 swab was not able to complete the testing procedure due to very narrow nasal passages of model 1. The asterisk indicates the significant difference calculated by multiple ANOVA statistical tests. Error bars represents SDs. C, Graph showing the relation of time and performance tests results for each of the tested swabs. Locations closer to the graph origin indicate faster and smoother test performance.

used for the testing. A broader range of ages and a larger number of patients would make this study more rigorous. The insertion tests were performed by 1 individual, a technologist with intimate knowledge of the nasopharyngeal anatomy. Testing by a medical professional who would routinely use such swabs in daily practice may change the results. Clinical validation was only performed on 2 adult volunteers and not on a larger number of pediatric subjects. All of these, however, are compromises we made for this emergency development.

Radiology departments have adopted 3D printers recently to provide prints for surgical planning and patient education and are uniquely positioned to produce $3 \mathrm{D}$-printed nasopharyngeal swabs during this pandemic. Additionally, they have unique access to volumetric imaging data to optimize and test swab designs. The methods described in this report demonstrate an effective collaboration between members of clinical and research departments in accelerating development and enabling creative patient-centric solutions.

Disclosures: Zbigniew Starosolski-UNRELATED: Patents (Planned, Pending or Issued): Texas Children's Hospital, Comments: submitted invention disclosure form to Texas Children's Hospital for a patent titled "Design of Pediatric 3D Printed Nasopharyngeal Swab on June 16, 2020; Stock/Stock Options: Alzeca Biosciences stock. Prasad Admane-RELATED: Grant: Baylor College of Medicine, Comments: Soicher Family Foundation*; RELATED: Patents (Planned,
Pending or Issued): Texas Children's Hospital, Comments: submitted invention disclosure form to Texas Children's Hospital for a patent titled "Design of Pediatric 3D Printed Nasopharyngeal Swab on June 16, 2020. Ananth Annapragada—RELATED: Grant: Soicher Family Foundation, Comments: to support work on bioprinting*; UNRELATED: Board Membership: Alzeca Biosciences; Consultancy: Alzeca Biosciences; Grants/Grants Pending: National Institutes of Health, Comments: I have several grants pending at National Institutes of Health*; Patents (Planned, Pending or Issued): numerous US and foreign patent applications, Comments: The full list can be obtained at https://patentscope. wipo.int/search/en/advancedSearch.jsf using search terms: IN: Ananth AND IN: Annapragada*; Royalties: University of Texas, Comments: royalties on patents licensed by the University of Texas to third parties; Stock/Stock Options: Sensulin, Alzeca Biosciences, Comments: I am a significant stockholder in Sensulin and Alzeca Biosciences; Other: Abbott Laboratories, Comments: I am a significant stockholder. *Money paid to the institution.

\section{REFERENCES}

1. Covidswab. http://github.com/rarnaout/Covideswab. Accessed April 1,2020

2. Centers for Disease Control and Prevention. CDC 2019-Novel Coronavirus (2019-nCoV) Real-Time RT-PCR. U.S. Food and Drug Administration emergency use authorization instructions for use. https://www.fda.gov/media/134922/download. Accessed July 17, 2020

3. Corman VM, Landt O, Kaiser M, et al. Detection of 2019 novel coronavirus (2019-nCoV) by real-time RT-PCR. Euro Surveill 2020;25:2000045 CrossRef Medline

4. Mullis KB, Faloona FA. Specific synthesis of DNA in vitro via a polymerase-catalyzed chain reaction. Methods Enzymol 1987;155:335-50 CrossRef Medline 